\title{
Impact Monitoring in Smart Composites Using Stabilization Controlled FBG Sensor System
}

\author{
H. J. Bang**a S. W. Park ${ }^{\mathrm{a}}$, D. H. Kimª , C. S. Hong ${ }^{\mathrm{a}}$, C. G. Kim ${ }^{\mathrm{a}}$ \\ ${ }^{a}$ Div. of Aerospace Engineering, Korea Advanced Institute of Science and Technology, \\ Daejeon 305-701, Korea
}

\begin{abstract}
Impact location monitoring is one of the major concerns of the smart health monitoring. For this application, multipoint ultrasonic sensors are to be employed. In this study, a multiplexed FBG sensor system with wide dynamic range was proposed and stabilization controlling system was also developed for the maintenance of maximum sensitivity of sensors. For the intensity demodulation system of FBG sensors, Fabry-Perot tunable filter (FP-TF) with $23.8 \mathrm{~nm}$ FSR (free spectral range) was used, which behaves as two separate filters between $1530 \sim 1560 \mathrm{~nm}$ range. Two FBG sensors were attached on the bottom side of the graphite/epoxy composite beam specimen, and low velocity impact tests were performed to detect the one-dimensional impact locations. Impact locations were calculated by the arrival time differences of the impact longitudinal waves acquired by the two FBGs. As a result, multiplexed in-line FBG sensors could detect the moment of impact precisely and found the impact locations with the average error of $1.32 \mathrm{~mm}$.
\end{abstract}

Keywords: optical fiber sensors, FBG sensors, smart structures, impact monitoring

\section{INTRODUCTION}

Low velocity impacts can cause invisible damages inside composite structures such as delaminations and matrix cracks. Because these composite fractures are hard to be found and degrade the load-bearing capacity of the structures, regular inspections are to be made in order to predict the remaining service life and reduce the danger of unexpected structural failure. The conventional NDE methods such as C-scan and X-ray, however, have possibility to miss out damages in hard to reach area [1] and also need to probe wide range of area in the structures to detect the damages in unknown impact locations. Thus, to improve the efficiency in damage diagnosis and guarantee the safety of composite structures also during operation, it is desirable to develop built-in health monitoring techniques as the concept of smart structure.

As a sensing part of the smart structures, fiber optic sensors (FOS) have shown the potential of the real-time health monitoring system. They can be easily embedded or attached to the structures without causing mechanical defects due to the small size and flexibility of the optical fiber. Also, they are immune to electromagnetic interference so that they are not affected by electrical noise from the environment. Recently, fiber optic sensors are expanding their application fields from strain measurement to fracture, vibration, temperature monitoring and simultaneous sensing of multiple parameters [2-4].

In order to detect impact locations, it is needed to develop the multiplexing technology for ultrasonic sensors. In this point of view fiber Bragg grating (FBG) sensors have advantages for this application since it can be applied to the wavelength division multiplexing method easily [5]. In the previous studies, many researchers have used a scanning filter for the multiplexing demodulator in the FBG sensor system [6] but in this case scanning frequency restricts the dynamic range of the FBG sensors. Thus they reluctantly reduced the number of sensors to sense the high frequency signals [7]. Another problem to be solved is that it was hard to maintain the sensitivity of the FBG ultrasonic sensors under the changing environments $[8,9]$ because the physical properties of optical components such as Fabry-Perot filter can easily influenced by external perturbations. Therefore, for the efficient and stable impact monitoring system, it is necessary to develop the stabilization controlled FBG sensor system capable of multiplexing with wide dynamic range. 
In this study, in order to find the impact locations in real-time, the multiplexed FBG sensor system with wide dynamic range was developed. A tunable Fabry-Perot filter with narrow free spectral range (FSR) was used to simplify the multiplexing demodulator for FBG ultrasonic sensors. Stabilization controlling system was also developed for the maintenance of the maximum sensitivity of sensors. And finally, we applied the proposed FBG sensor system to onedimensional impact location monitoring tests with a graphite/epoxy composite beam specimen.

\section{FBG SENSOR SYSTEM}

\subsection{Theory of measurement}

A fiber Bragg grating is a periodic perturbation of refractive index formed in the core of an optical fiber by using an intensive UV laser. If the broadband light sheds on the Bragg grating, it only reflects the specific wavelength component and works like a narrowband reflecting filter [5], which is described by the Bragg condition in Equation (1).

$$
\lambda_{B}=2 n_{e} \Lambda
$$

Where $\lambda_{B}$ is the Bragg wavelength of FBG, $n_{e}$ is the effective index of the fiber core, and $\Lambda$ is the grating period.

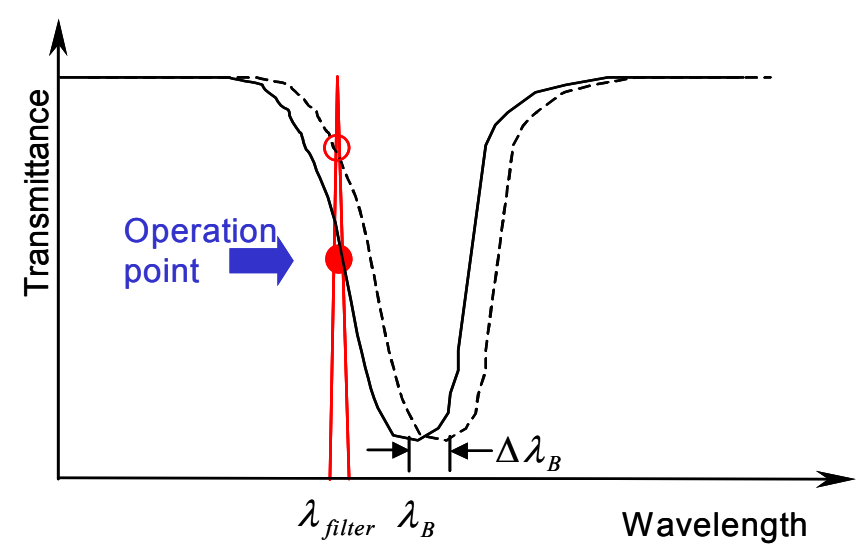

Fig. 1 Principle of interferometric demodulation for the FBG ultrasonic sensor system.
Because the effective index and grating period are the functions of temperature and strain, Bragg wavelength is changed when temperature changes or mechanical strain is applied to the FBG sensor.

Figure. 1 shows the concept of demodulation method used in the FBG acoustic sensor system. As shown in the figure, placing the narrow band filter at the operation point of the FBG spectrum, output intensity from the filter is being changed in case the Bragg wavelength is shifted due to external perturbations. If we assume that the Bragg wavelength linearly changes as the applied strain and the slope of the FBG spectrum near the operation point is constant, the output intensity of the filter can be expressed as Equation (2) [9].

$$
I_{\text {out }}=I_{\text {in }}\left(T_{0}+S \zeta_{F}\right)
$$

Where $\zeta_{F}$ is instantaneous strain applied to the FBG, $T_{0}$ and $S$ are the initial transmittance and slope at the operation point, respectively. From the above equation, within the linear region of the FBG spectrum strain perturbations such as AE signals are displayed as the intensity variations of the output light.

\subsection{Multiplexing method with wide dynamic range}

Because the Fabry-Perot demodulator for the FBG sensors is a multi-beam interferometer having a mirror cavity, the transmittance of the output beam can be expressed as the following Equation [5].

$$
\frac{I_{t}}{I_{i}}=\frac{1}{1+\left(\frac{2 F}{\pi} \sin \frac{2 \pi n d}{\lambda}\right)^{2}}
$$

Where $I_{i}$ and $I_{t}$ are the intensities of the input and output lights of the Fabry-Perot demodulator, respectively, and $F$ is the finesse, $n$ is the refractive index and $d$ is the length of the Fabry-Perot cavity. The right term of Equation (3), containing Airy function, is a periodic function and has the maximum values when $\sin (2 \pi n d / \lambda)$ becomes zero. Thus, the 
filtering wavelength of the Fabry-Perot demodulator has the regular wavelength interval, FSR(free spectral range), as Equation (4) [5].

$$
\lambda=\frac{2 n d}{N}
$$

Because a FBG sensor only reflects the Bragg wavelength of confined bandwidth, if we use the FBG sensors having different reflecting wavelengths, we can detect the distributed physical properties using the multi-point sensing ability of the in-line FBG sensor array.

Matching the Bragg wavelength to the filtering wavelength of the Fabry-Perot demodulator with narrow FSR, plural FBG sensors can be simultaneously demodulated using a single demodulator. This demodulation scheme plays an equivalent role as the employment of several filters with the same number of FBG sensors and also provides multiplexing ability without causing the losses in dynamic range. By using the Fabry-Perot filter with narrow FSR, the demodulation system can be downsized and simplified for the case of demanding the multiplexing as well as sensing of high frequency signals.

\subsection{Stabilization controlling unit}

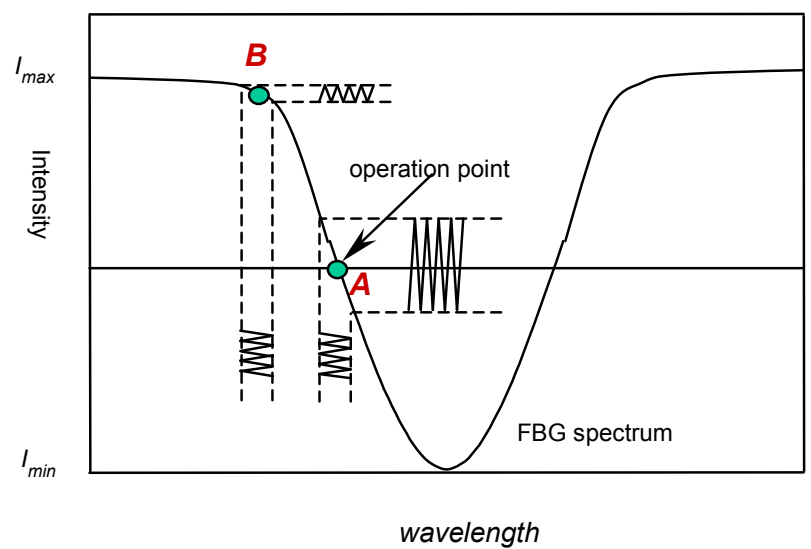

Fig. 2 Fade-out problem : signal intensity variation due to the slope of the FBG spectrum.
Either temperature or quasi-static strain, however, can influence the optical properties of the Fabry-Perot filter and laser. As shown in Fig.2 if the filter wavelength moves from the point A to point B intensity of the output signal becomes smaller for the same input. This is called fade-out problem that causes sharp decrease in sensitivity of the FBG ultrasonic sensors. Thus in order to maintain the maximum sensitivity of the FBG ultrasonic sensors, the filtering wavelength of the demodulator should be fixed to the operation point of the FBG spectrum where the slope is the steepest. In this study, controlling the filtering position using the closed loop controller with the tunable Fabry-Perot filter, we can fix the wavelength of the demodulator at the operation point, the maximum sensitive region of the FBG spectrum. The active control system used for the sensitivity stabilization consists of a tunable Fabry-Perot filter, WDM (wavelength division multiplexer) and I/O board (PCI-6110E, National Instrument). Figure. 3 shows the schematic diagram of the FBG acoustic sensor system equipped with the stabilization controlling unit. Sensor signals are divided by the WDM and fed into the I/O board of the signal-processing computer through the photo detector. In the signal processor, the intensity level of the input signal is compared with that of

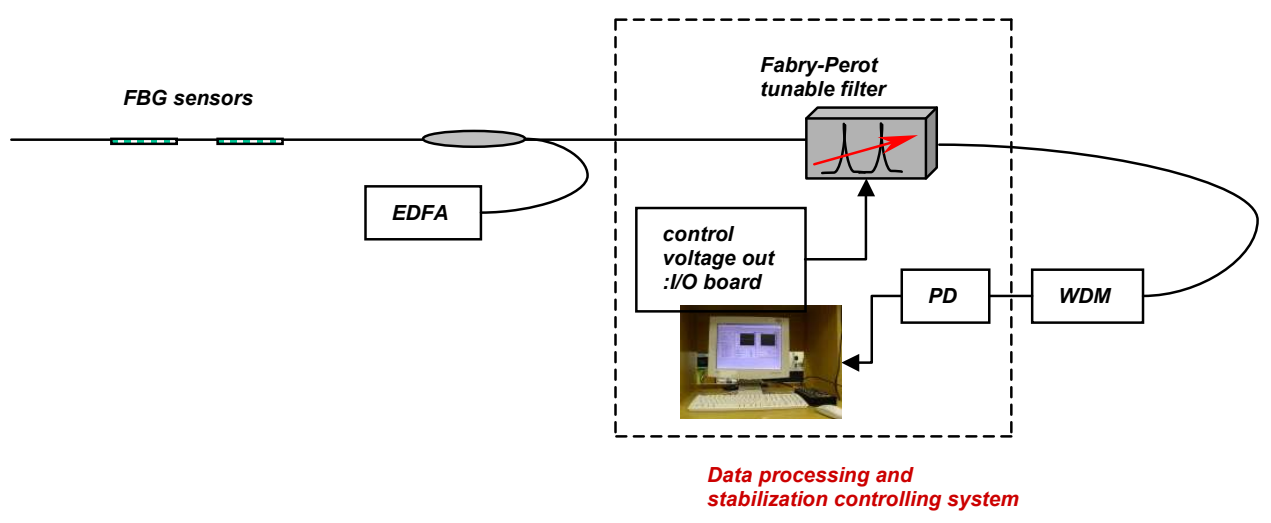

Fig. 3 Closed-loop stabilization controlling FBG sensor system. 
reference value in a comparator after low pass filtering. The difference between them is compensated through the wavelength shift of the light that enters into the sensor. Consequently these continuous processes form the closed loop circuit which is shown in the dotted box in Fig. 3.

If the signal processor has enough calculation speed to compensate the wavelength shifts, not associated with the expected signals, the sensitivity of the FBG sensors are to be maintained regardless of the environmental influences. Thus, low frequency fluctuations such as thermal expansion are removed from the original signal and high frequency components of stress waves are separated and acquired. In this study, a software based signal processing system was developed using GUI interface considering the flexibility of the future expansion to the multi-channel FBG sensor system.

\subsection{Experimental setup}

\section{EXPERIMENTS}

Using the two FBG sensors, low velocity impact monitoring tests were performed for a composite beam specimen. The two FBGs have the same gage length of $5 \mathrm{~mm}$, and the Bragg wavelengths of the sensors are $1532.4 \mathrm{~nm}$ and 1556.2 $\mathrm{nm}$ for FBG1 and FBG2, respectively. This wavelength spacing of the FBGs is designed to fit the interval of the filtering wavelength of the tunable Fabry-Perot demodulator.

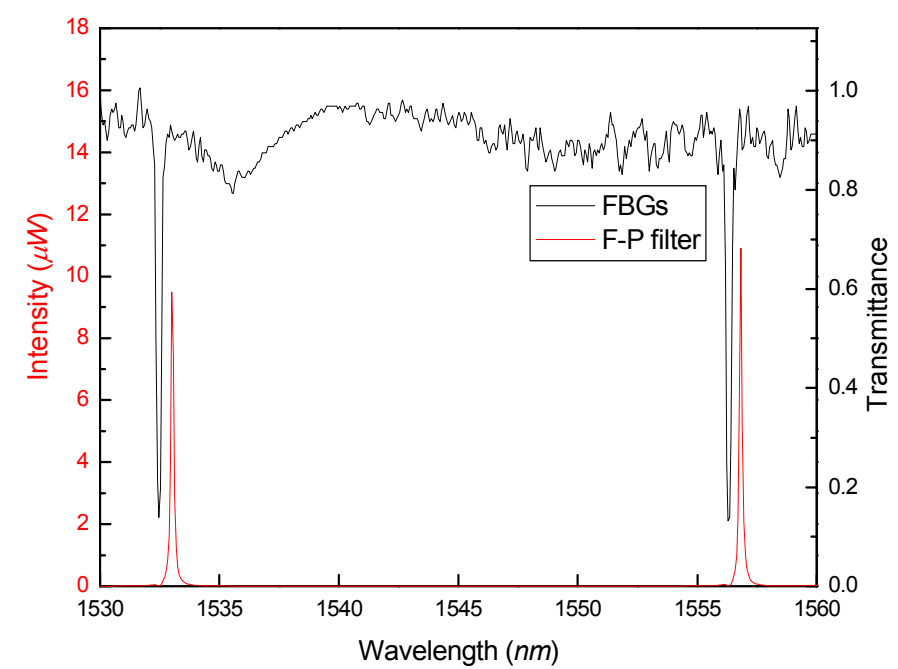

Fig. 4 Spectrum of FBGs and tunable Fabry-Perot filter.

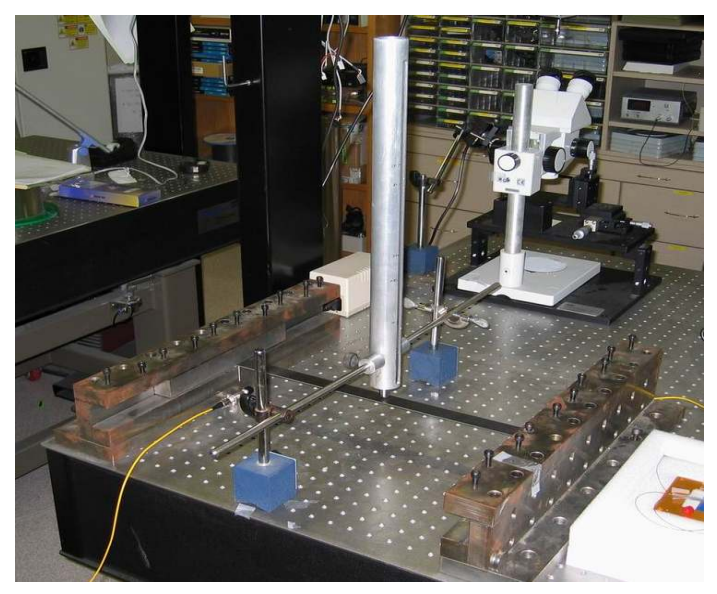

Fig. 5 Experimental setup for impact location monitoring.

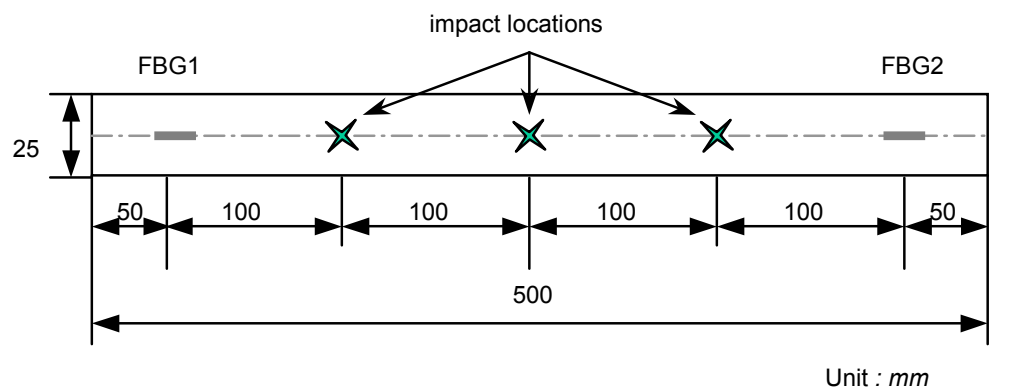

Fig. 6 Schematic figure of composite beam specimen for impact monitoring test. 
Figure. 4 shows the wavelength spectrum of the Fabry-Perot demodulator and two FBG sensors. The filtering wavelengths of the demodulator were tuned to the operation points of the FBG spectrum before the experiments. And, the stabilization-controlling system continuously readjusted these filtering locations through the experiments so that the maximum sensitivity of the FBG sensor was possibly maintained. The overall view of experimental setup is presented in Fig. 5. Impact loads were applied by dropping the guided weight, and impact energy could be changed by controlling the mass of the weight and dropping height.

As shown in Fig. 6, the composite beam specimen had the dimension of $500 \mathrm{~mm} \times 25 \mathrm{~mm} \times 1.6 \mathrm{~mm}(L \times W \times T)$ and each opposite end was clamped to the fixing jig. Two FBG sensors were attached to the bottom surface of the specimen and PZT sensors were also bonded on the opposite side for the comparison with the FBG sensors. We used the response of FBG1 as a trigger signal. In this experiment, $0.15 \mathrm{~J}$ of impact energy was inflicted on the central regions of the specimen. Three impact locations were selected as $100 \mathrm{~mm}, 200 \mathrm{~mm}$ and $300 \mathrm{~mm}$ away from the FBG1 along the longitudinal direction of the beam, and the assumption of one-dimensional wave propagation was applied to the signal processing for the impact location detection.

\subsection{Results and discussion}

The stress wave signal produced by the impact load can be divided into two waves by their propagation speeds, longitudinal waves and transverse waves. Longitudinal waves travel relatively faster than transverse waves and have regular reproducibility. But the transverse waves have low frequency components with irregularity which are dominantly below $20 \mathrm{kHz}$.

Figure. 7 is the comparison of the impact stress wave signals and their short-time Fourier transformed (STFT) results detected by FBG and PZT when an impact load was applied to the center of the specimen. The upper figures show the time-domain responses and the lower ones indicate the time-frequency signals of STFT. In time domain signals, longitudinal waves lasted about $0.3 \mathrm{~ms}$ from the beginning of the impact signals and show the similar profile between the FBG and PZT sensor signals. STFT results also show the same signal characteristics between the two sensors in the longitudinal wave region, $0.8 \mathrm{~ms} \sim 1.1 \mathrm{~ms}$, and the signal level in the frequency domain shows almost even distribution in the frequency range of $1 \sim 40 \mathrm{kHz}$ in it. After $1.2 \mathrm{~ms}$ in Fig.7, the transverse waves followed the longitudinal waves having higher energy level in audio frequency range. These results confirm that FBG sensors can detect the stress wave signals induced by the impacts and the signal characteristics of the longitudinal waves of FBG are identical with those of PZT.
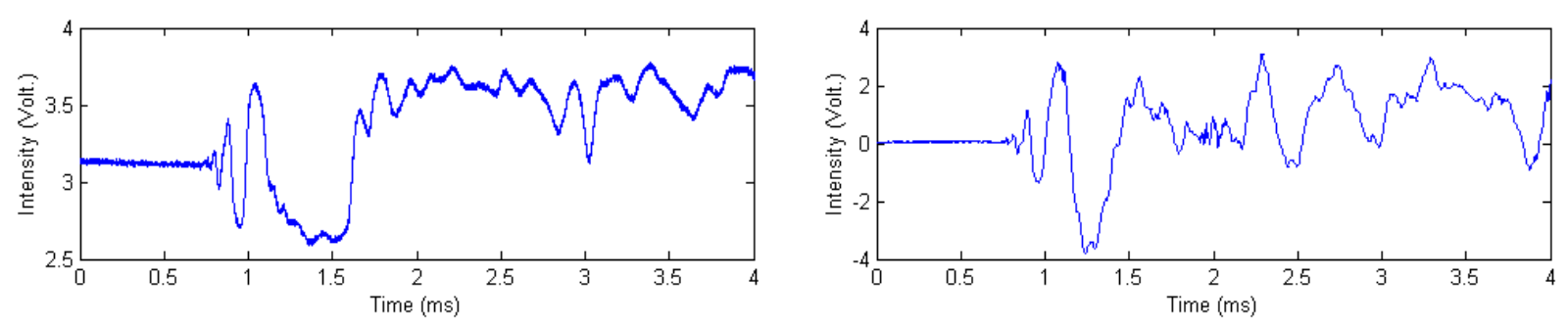

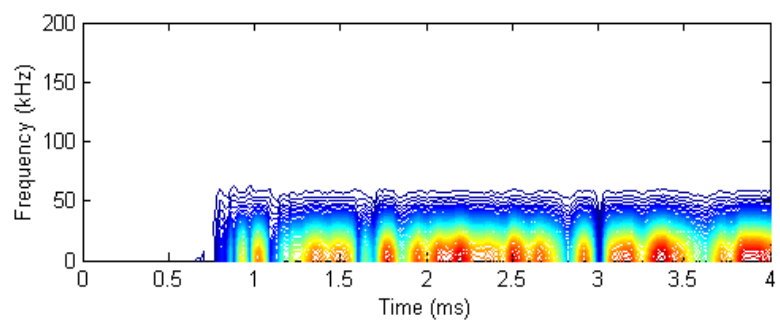

(a) FBG signal

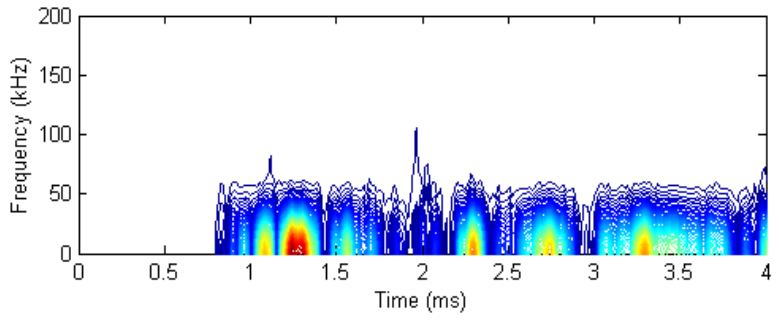

(b) PZT signal

Fig. 7 Comparison of stress wave signals and its STFT induced by impact. 
In this study, the arrival time difference of the longitudinal waves between the two FBG sensors were used to detect the impact location because the longitudinal waves are reproducible for the same impact loads and they are not much influenced by boundary conditions [1].

In $\left[0^{\circ} / 45^{\circ} /-45^{\circ} / 90^{\circ}\right]_{2 S}$ composite plates, the longitudinal waves have the frequency range of $1 \sim 40 \mathrm{kHz}$. If we assume that the propagation speed, $v$, is constant along the longitudinal direction of the composite beam specimen, mathematical formulation of the impact location can be expressed as Equation (5) [10].

$$
\Delta t_{i j} v=\sqrt{\left(x_{i}-x\right)^{2}-\left(y_{i}-y\right)^{2}}-\sqrt{\left(x_{j}-x\right)^{2}-\left(y_{j}-y\right)^{2}}
$$

where $\left(x_{i}, y_{i}\right)$ and $\left(x_{j}, y_{j}\right)$ are the positions of the FBG1 and FBG2, respectively, and $(x, y)$ indicates the location of the impact. The symbol, $v$, stands for the propagation speed of the longitudinal waves and it is calculated by the time difference of the arriving waves, $\Delta t_{i j}$, between the sensors. In the experiments, propagation speed, $v$, was measured as $0.75 \mathrm{~km} / \mathrm{s}$ in the $\left[0^{\circ} / 45^{\circ} /-45^{\circ} / 90^{\circ}\right]_{2 S}$ graphite/epoxy composite specimen.

Figure. 8(a) shows the AE signals detected by the two FBGs for the $1^{\text {st }}$ impact location, $100 \mathrm{~mm}$ away from the FBG1. The longitudinal waves of the impact signals lasted about $0.3 \mathrm{~ms}$ before the transverse waves followed. Time differences

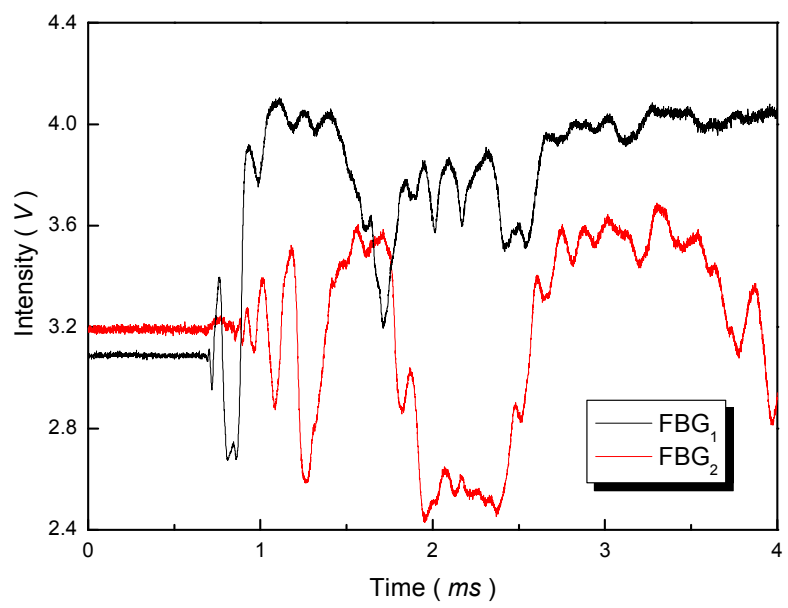

(a) impact signals at $100 \mathrm{~mm}$

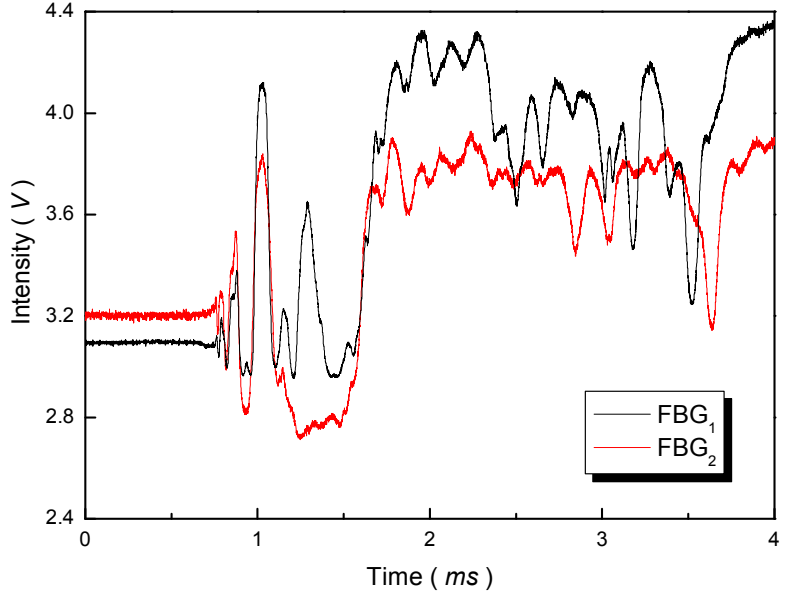

(b) impact signals at $200 \mathrm{~mm}$ (center)

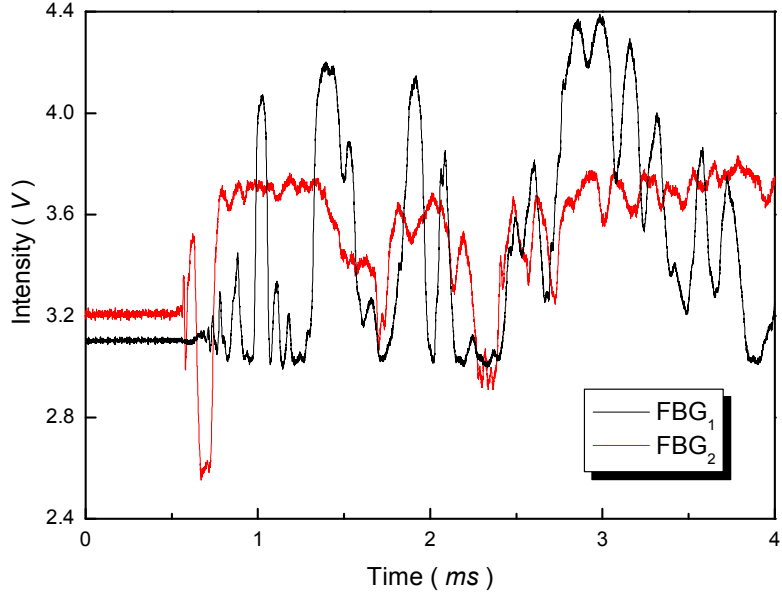

(c) impact signals at $300 \mathrm{~mm}$

Fig. 8 Stress wave signals detected by the FBG sensors of the three impact locations. 
of the wave propagation are derived from the locations of the peaks of longitudinal waves. As a result, signal processing estimated the impact location as $100.24 \mathrm{~mm}$ from the position of FBG1 and the average error was $1.40 \mathrm{~mm}$. Figure. 8(b) is the impact signals generated from the center of the specimen, $2^{\text {nd }}$ impact location, at the distance of $200 \mathrm{~mm}$ away from the FBG1. In this case, because the distances between the impact location and two FBG sensors are the same, signal shapes in longitudinal waves from the two FBGs are similar and there is no time difference. The detected impact location was $200.22 \mathrm{~mm}$ and average error was $1.56 \mathrm{~mm}$. Figure. 8(c) is the result of $3^{\text {rd }}$ impact location, and calculated impact location was $300.39 \mathrm{~mm}$ and average error was $0.96 \mathrm{~mm}$. Comparing the signal magnitudes of the results in Fig. 8, it was found that intensity levels of the output signals had little difference to each other, because the sensitivity of the sensors was stabilized through the experiments.

Above experimental results are summarized in Table 1. As the results of impact tests, the maximum error of these results was $4.54 \mathrm{~mm}$, and the average location error for all sets was $1.32 \mathrm{~mm}$ for the detection of the impact locations. In conclusion, we could accurately find the impact locations in the composite specimens using the stabilization controlled FBG sensor system, and the system showed enough sensitivity to detect the subtle longitudinal waves from acoustic signals.

Table 1. Result of impact location monitoring.

\begin{tabular}{|c||c|c|c|}
\hline Actual location $(\mathbf{m m})$ & $\mathbf{1 0 0}$ & $\mathbf{2 0 0}$ & $\mathbf{3 0 0}$ \\
\hline \hline $\begin{array}{c}\text { Average detected location }(\mathrm{mm}) \\
\text { Standard } \\
\text { deviation }\end{array}$ & 100.24 & 200.22 & 300.39 \\
\hline Mean error $(\mathrm{mm})$ & 0.0016 & 0.0022 & 0.0011 \\
\hline Maximum error $(\mathrm{mm})$ & 1.40 & 1.56 & 0.96 \\
\hline Total mean error $(\mathbf{m m})$ & 3.61 & 4.54 & 2.97 \\
\hline Total maximum error $(\mathbf{m m})$ & $\mathbf{3}$ & $\mathbf{4 . 3 2}$ \\
\hline
\end{tabular}

\section{CONCLUSION}

In this study, one-dimensional impact monitoring tests were performed as an application of the FBG sensor system of wide dynamic range. A multiplexing demodulator with wide dynamic range was used to detect the ultrasonic longitudinal waves of impact signals, and stabilization-controlling unit was also developed for the maintenance of maximum sensitivity of FBG sensors. And the proposed FBG sensor system was applied to the impact tests of the graphite/epoxy composite beam specimen.

As the result of the impact test, it is confirmed that FBG sensors precisely can detect the stress wave signals induced by the impacts and the signal characteristics of the longitudinal waves of FBG are identical with those of PZT. And multiplexed in-line FBG sensors found the impact locations with the average error of $1.32 \mathrm{~mm}$ and the maximum error of $4.54 \mathrm{~mm}$. Consequently, we can conclude that above FBG system has the wide dynamic range enough to sense the ultrasonic waves with stable performance and the potential to be applied to the impact monitoring and damage assessment in composite structures.

\section{ACKNOWLEDGEMENTS}

The authors would like to thank the Agency for Defense Development, Korea, for the financial support (ADD-03-0502). 


\section{REFERENCES}

1. D.U. Sung, J. H. Oh, C. G. Kim and C. S. Hong, "Impact Monitoring of Smart Composite Laminates Using Neural Networks and Wavelet Analysis" Journal of Intelligent Material Systems and Structures, Vol. 11, 2000, pp. 180190.

2. J. W. Park, C. Y. Ryu, H. K. Kang and C. S. Hong, "Detection of Buckling and Crack Growth in the Delaminated Composites Using Fiber Optic Sensor," Journal of Composite Materials, Vol. 34, No. 19, 2000, pp. 1602-1623.

3. H. K. Kang, H. J. Bang, C. S. Hong and C. G. Kim, "Simultaneous Measurement of Strain, Temperature, and Vibration Using Fiber Optic Sensor," Measurement Science and Technology, Vol. 13, No. 8, 2002, pp. 1191-1196.

4. A. D. Kersey, T. A. Berkoff and W. W. Morey, "Multiplexed Fiber Bragg Grating Strain Sensor System with a Fiber Fabry-Perot Wavelength Filter," Optics Letters, Vol. 18, No. 16, 1993, pp. 1370-1372.

5. C. Y. Ryu and C. S. Hong, "Development of Fiber Bragg Grating Sensor System Using Wavelength-Swept Fiber Laser, "Smart Materials \& Structures, Vol. 11, No.3, 2002, pp. 468 - 473.

6. M. D. Todd, G. A. Johnson and B. L. Althouse, " A Novel Bragg Grating Sensor Interrogation System Utilizing a Scanning Filter, a Mach-Zehnder Interferometer and a 33 coupler," Measurement Science and Technology, Vol. 12, 2001, pp. 771-777.

7. D. C. Betz, G. Thursby, B. Culshaw and W. J. Staszewski, "Acousto-Utrasonic Sensing Using Fiber Bragg Gratings," Smart Materials and Structures, Vol. 12, 2003, pp. 122-128.

8. J. F. Dorighi, S. Krishnaswamy and J. D. Achenbach, "Stabilization of an Embedded Fiber Optic Fabry-Perot Sensor for Untrasound Detection," IEEE Transactions on Ultrasonics, Ferroelectrics, and Frequency Control, Vol. 42, No. 5, 1995, pp. 820-824.

9. N. Takahashi, K. Yoshimura and S. Takahashi, "Detection of Ultrasonic Mechanical Vibration of a Solid Using Fiber Bragg Grating," Japanese Journal of Applied Physics, Vol. 39, 2000, pp. 3134-3138.

10. J.A. Greene, T.A. Tran, A. Murphy and R.O. Claus et al., "Optical Fiber Sensing Technique for Impact Detection and Location in Composites and Metal Specimens," Smart Materials and Structures, Vol. 4, 1995, pp. 93-99. 